\title{
A Patient with Pseudomonas aeruginosa vertebral osteomyelitis presenting with back pain on a telephonic encounter due to COVID-19
}

\section{Eugene F Sun* \\ Presbyterian Medical Services, New Mexico}

\section{Introduction}

The COVID-19 National Public Health Emergency has resulted in a high proportion of office visits for healthcare being changed to telephonic or video visits. Assessing patients with unknown, potentially serious conditions is challenging. There may be timeliness concerns with diagnostic labs or imaging due to scheduling issues or managed care organization $(\mathrm{MCO})$ prior authorization requirements.

\section{Case presentation}

A 59 year old male had a telephonic visit for complaint of back pain for one month. Due to the COVID-19 Health Emergency the majority of patient visits at this Federally Qualified Health Center had been transitioned to telephonic visits.

A month prior he had a serious illness with fever, chills, nausea, and vomiting that resolved. He did not seek medical care then due to no health insurance coverage. He subsequently developed severe right upper back and lateral chest pain which was worse with coughing and deep inspiration. There was no sputum production or shortness of breath. Pain increased with lying on right side. Around the time he presented for care, he became enrolled in a managed Medicaid plan. weeks.

Patient denied history of diabetes but had nocturia for past few

No exam was possible given this was a telephonic visit.

Sodium was $138 \mathrm{mmol} / \mathrm{L}$, potassium $3.8 \mathrm{mmol} / \mathrm{L}$, chloride $106 \mathrm{mmol} / \mathrm{L}$, bicarbonate $24 \mathrm{mmol} / \mathrm{L}$, blood urea nitrogen $9 \mathrm{mg} /$ $\mathrm{dL}$, creatinine $0.99 \mathrm{mg} / \mathrm{dL}$, alkaline phosphatase $77 \mathrm{U} / \mathrm{L}$, alanine aminotransferase $23 \mathrm{U} / \mathrm{L}$, aspartate transaminase $13 \mathrm{U} / \mathrm{L}$, total bilirubin $0.3 \mathrm{mg} / \mathrm{dL}$, calcium $9.5 \mathrm{mg} / \mathrm{dL}$, albumin $3.0 \mathrm{gm} / \mathrm{dL}$ glucose $88 \mathrm{mg} / \mathrm{dL}$. Serum globulin was elevated at $5.6 \mathrm{gm} / \mathrm{dL}$. White blood cells $10.0 \mathrm{x}$ $10^{3} / \mu \mathrm{L}$, Hematocrit $39 \%$, hemoglobin $12.5 \mathrm{gm} / \mathrm{dL}$, platelets $491 \times 10^{3} /$ $\mu \mathrm{L}$. No left shift reported. Urinalysis was cloudy, many bacteria, small amount blood, large leukocyte esterase, positive nitrite, protein 30 , red blood cells 42 and white blood cells greater than 150 .

Because of the elevated globulin level a serum electrophoresis was done which showed a polyclonal increase in gamma globulin fraction. C-reactive protein was $3.7 \mathrm{mg} / \mathrm{dL}$.

A two view PA lateral chest $\mathrm{x}$-ray noted no acute cardiopulmonary process. X-ray of right ribs did not show any fracture but noted blunting of the right costophrenic angle.

Urine culture had greater than $100,000 \mathrm{cfu} / \mathrm{mL}$ each of two different strains of Pseudomonas aeruginosa. The strains were pan-sensitive.
Due to reported penicillin allergy, the patient was started on oral ciprofloxacin $500 \mathrm{mg}$ twice a day due for an initial seven day course. Patient stated that his symptoms improved with nocturia resolving. The back pain improved slightly but persisted. Because of the persistent pain, ciprofloxacin was continued an additional 7 days.

To evaluate the etiology of the Pseudomonos aeruginosa UTI a renal ultrasound was done 15 days after initial presentation. There was no evidence of hydronephrosis on either side and no obvious calcification. No perirenal fluid collections were seen but it was noted that ultrasound is relatively insensitive for that. Post void residual was $23 \mathrm{~mL}$. There was no detected abnormality and a CT was recommended if there was concern for obstruction or localized renal infection.

Patient was seen face to face in the office after the renal ultrasound. Patient was afebrile with normal vital signs but with persistent, moderately severe right upper back pain. On exam there was pain to palpation and percussion right upper posterior ribs from near midline to inferior to scapula. A repeat urine culture had no growth.

A chest and abdominal CT scan were ordered and prior authorization request was submitted to the $\mathrm{MCO}$. The vendor contracted by the MCO denied authorization, and a peer to peer discussion was done during which the Pseudomonas aeruginosa UTI was discussed. Based on that the CT was approved. By the time the scan was scheduled and performed, 4 weeks had elapsed from the date of the ultrasound, and 6 weeks from the time of initial presentation.

The CT scan noted minimal delayed excretion of the right kidney with right lower pole $0.3 \mathrm{~cm}$ calyceal calcification. Mild to moderate hydronephrosis with a $0.6 \mathrm{~cm}$ calcification/stone in the right proximal and a $0.3 \mathrm{~cm}$ calcification in the right mid ureter were noted.

Also noted were complete destructive changes of C6 inferior endplate and C7 superior endplate with soft tissue thickening extending into the anterior paravertebral soft tissues, possibly secondary to infectious discitis. Magnetic resonance imaging of thoracic spine recommended. Based on that finding, the patient was immediately restarted on ciprofloxacin 1000mg orally bid, and MRI ordered.

${ }^{\star}$ Correspondence to: Eugene F Sun, Presbyterian Medical Services, New Mexico, E-mail: eugene.sun@pmsnm.org

Key words: COVID-19, Pseudomonas aeruginosa, vertebral osteomyelitis, managed care, prior authorization, pandemic

Received: June 08, 2020; Accepted: June 25, 2020; Published: June 29, 2020 
The MRI was completed 6 days after CT, 7 weeks from initial presentation and showed disk space infection and adjacent vertebral body osteomyelitis at the T5-6 level with bony destruction of endplates. T3, T4 and T7 vertebral bodies also showed osteomyelitis with abnormally increased T2 signal and enhancement of the T8-9 intervertebral disc. No mention was made of the C6-7 area in the MRI report.

Case was discussed with neurosurgeon and decision made to send patient to hospital emergency department for admission. Patient was admitted the same day of MRI, 7 weeks from initial telephone presentation.

Patient was hospitalized and treated with IV antibiotics and underwent cystoureteroscopy with right laser lithotripsy and ureteral stent placement. Neurosurgery evaluation indicated discitis T5-T6 with collapse and kyphosis, abscess encircling the cord and developing imminent bony collapse. MRI of cervical spine did not indicate any infectious process in the cervical spine.

Patient underwent T5/6 corpectomy with placement of expandable cage graft, T2-T9 posterior spinal fusion with pedicle screw and rod construct, and T5-T6 laminectomy with decompression of spinal cord and washout.

The apparent discrepancy in the initial outpatient CT report of infectious discitis of $\mathrm{C} 6$ and $\mathrm{C} 7$ and the inpatient hospital cervical MRI which did not mention any issues with $\mathrm{C} 6$ and $\mathrm{C} 7$ was not reconciled.

Patient was discharged on day 14 with six weeks IV antibiotics and appropriate follow up.

\section{Discussion}

Vertebral osteomyelitis (spondylodiscitis) is an uncommon condition. It has been reported that the estimated annual incidence is approximately 2.4 cases per 100,000 [1], though other reports indicate a rate as high as 5.4 per 100,000 [2]. The lumbosacral spine is involved most frequently followed by the thoracic then cervical spine.

Risk factors include diabetes, injection drug use, prior spine surgery, and infective endocarditis among others. In this case the patient had none of those factors. Pathogens can include $S$ aureus, pyogenic streptococci, enteric gram negative bacilli and $P$. aeruginosa. Spondylodiscitis due to $P$. aeruginosa is very rare and in case reports have been attributed to injection drug use, pneumonia, diabetes, and dental procedures.
In this case the patient developed a $P$. aeruginosa UTI as a result of the renal calcification and obstruction. He developed a severe illness, possibly including pyelonephritis and urosepsis but did not seek medical care due to lack of health insurance. During this time the thoracic vertebrae were seeded, presumably via Batson's venous plexus, causing the osteomyelitis that led to his seeking care for back pain.

It is widely reported and believed that the COVID-19 national health emergency has created significant barriers for patients to access and receive care. Gupta [3] reports that routine outpatient visits have decreased by 30 percent. As in this case, patients may lack access to health insurance and be reluctant to incur potentially high out of pocket costs, until their condition becomes more severe and unbearable. Patients may also be reluctant to risk possible exposure to the virus at a medical office or facility. Compounding those issues, there may be decreased or limited scheduling access to outpatient office or remote visits.

Once under care, structural barriers including decreased availability of advanced diagnostic imaging due to constraints from the COVID-19 pandemic and MCO prior authorization procedures may further slow or impede appropriate care.

\section{Conclusion}

As states proceed with phased reopening, and as PPE supplies permit, medical offices and facilities will expand the services they offer. It is likely that telephone visits and video visits will persist at a higher level than pre-pandemic. While those modalities can provide preventive care and screening, this case highlights the need for continued vigilance in evaluating symptomatic patients who present remotely to ensure that potentially serious conditions are identified, evaluated, and treated as timely as possible.

\section{References}

1. Wang CC (2012) Pseudomonas aeruginosa costovertebral arthritis in association with spontaneous cervical spondylodiscitis and epidural absesses in the elderly. J Clin Gerontol Geriatr 3: 82-86.

2. Issa K (2018) The epidemiology of vertebral osteomyelitis in the United States from 1998 to 2013. Clin Spine Surg 31: E102.

3. Gupta R (2020) Collateral damage occurs when doctors and patients wear 'Covid-19 blinders'. Statnews May 4, 2020

Copyright: (C2020 Sun EF. This is an open-access article distributed under the terms of the Creative Commons Attribution License, which permits unrestricted use, distribution, and reproduction in any medium, provided the original author and source are credited. 